\title{
5 Ausschluss der Auskunftserteilung zum Wohl der betroffenen Person
}

Das folgende Kapitel beantwortet die Forschungsfrage 4.5:

Der Bundesrat hat in seinem Beschluss zum DSAnpUG-EU (Vgl. Ziff. 27 Lit. d), BR-Drucksache 110/17 v. 10.03.2017) für die Behandlung im weiteren Gesetzgebungsverfahren die Frage aufgeworfen, inwieweit ein Ausschluss der Auskunftserteilung neben den in $\$ 27$ BDSG-Egenannten Voraussetzungen nach objektiven Kriterien auch aus therapeutischen sowie ethischen Erwägungsgründen zum Wohl der betroffenen Person möglich sein sollte. Prüfen Sie bitte auf welcherverfassungs- und europarechtlichen Grundlage solche Ausnahmen von den Auskunftsrechten für wissenschaftliche Forschungsvorhaben vorgenommen werden können.

Dem Recht auf Auskunft kommt eine zentrale Rolle im Datenschutzrecht zu. Nur über das Auskunftsrecht kann der Betroffene tatsächlich überprüfen, „wer was wann und bei welcher Gelegenheit" über ihn weiß. ${ }^{365}$ Dennoch wird das Auskunftsrecht weder im geltenden noch im neuen Datenschutzrecht schrankenlos gewährt.

365 BVerfGE 65, 1 (43). 


\subsection{Auskunftserteilung nach Art. 15 DSGVO}

Das Recht auf Auskunft in Art. 15 DSGVO ist im Wesentlichen ${ }^{366}$ deckungsgleich mit seinem Vorgänger aus der Datenschutzrichtlinie und seiner Umsetzung im bisherigen Bundesdatenschutzgesetz. Das Auskunftsrecht ist zweitstufig aufgebaut. Auf Antrag der betroffenen Person ist der Verantwortliche verpflichtet, diese darüber $\mathrm{zu}$ informieren, ob er personenbezogene Daten der betroffenen Person verarbeitet. Ist dies der Fall, so sind der betroffenen Person bestimmte Informationen über die Umstände der Verarbeitung und ihrer Rechte gegenüber dem Verantwortlichen ${ }^{367}$ zur Verfügung zu stellen. Hierzu findet sich in Art. 15 Abs. 1 Hs. 2 lit. a bis h DSGVO ein abschließender Katalog. Werden personenbezogene Daten an ein Drittland oder an eine internationale Organisation übermittelt, treten nach Art. 15 Abs. 2 DSGVO weitere Informationen zu den geeigneten Garantien nach Art. 46 DSGVO hinzu. Nach Art. 15 Abs. 3 DSGVO ist der betroffenen Person zudem eine Kopie der sie betreffenden personenbezogenen Daten zur Verfügung zu stellen. Die Rechte und Freiheiten anderer Personen dürfen durch das Recht nach Abs. 3 nicht beeinträchtigt werden. ${ }^{368}$ Erwägungsgrund 63 DSGVO nennt hier beispielhaft Geschäftsgeheimnisse und Rechte des geistigen Eigentums. Die Feststellung, welche Rechte überwiegen, bedarf einer Abwägung im Einzelfall.

Das Recht auf Auskunft bildet die Basis für die Überprüfung der Rechtmäßigkeit der Verarbeitung durch den Betroffenen. ${ }^{369}$ Ohne Auskunftsrecht sind die ihm nachfolgenden Rechte auf Berichtigung, Löschung, Einschränkung der Verarbeitung, Datenübertragbarkeit und Widerspruch oftmals faktisch nicht wahrnehmbar.

Erwägungsgrund $6_{3}$ DSGVO erläutert, dass die Ausübung des Auskunftsrechts auf angemessene Abstände beschränkt sein soll. Gesundheitsbezogene Daten sind ausdrücklich vom Auskunftsrecht erfasst.

Die Form der Bereitstellung ist in Art. 15 Abs. 3 DSGVO geregelt. Wird der Antrag auf Auskunft elektronisch gestellt, so ist bei der Bereitstellung ein „gängiges elektronisches Format“ zu wählen. Dem Antragsteller steht es aber frei, ${ }^{370}$ ein anderes Format zu verlangen, sofern in der Wahl des Formats kein Missbrauch des Auskunftsrechts liegt. ${ }^{371}$ Erwägungsgrund 63 DSGVO fordert ferner die Einrichtung eines Fernzugangs.

366 S. zu den Abweichungen im Detail Hohmann/Miedzianowski, in: Roßnagel 2018, §4 Rn. 7, 14.

367 Namentlich die Rechte aus Art. 16, 17, 18, 21 und 79 DSGVO.

368 Art. 15 Abs. 4 DSGVO.

369 Ehmann, in: Ehmann/Selmayr, Art. 15 Rn. 1.

370 Ob teilweise (so Bäcker, in: Kühling/Buchner, Art. 15 Rn. 31) oder vollumfänglich ist dabei umstritten.

371 Franck, in: Gola, Art. 15 Rn. 23, 27. Bei exzessiven Anträgen kann der Verantwortliche Nach Art. 12 Abs. 5 Satz 2 DSGVO sich wahlweise weigern, tätig zu werden, oder ein angemessenes Entgelt verlangen. 
Die weiteren Modalitäten der Ausübung des Auskunftsrechts finden sich in Art. 12 DSGVO. So hat die Mitteilung gemäß Art. 15 DSGVO durch den Verantwortlichen etwa nach Art. 12 Abs. 1 Satz 1 DSGVO „in präziser, transparenter, verständlicher und leicht zugänglicher Form in einer klaren und einfachen Sprache“ zu erfolgen. ${ }^{372}$ Die Auskunft muss nach Art. 12 Abs. 5 DSGVO unentgeltlich erfolgen.

\subsection{Auskunftserteilung nach Art. 8 Abs. 2 Satz 2 GRCh}

Das Recht auf Auskunft aus Art. 15 DSGVO ergibt sich unmittelbar aus Art. 8 Abs. 2 Satz 2 GRCh, der ein solches Recht primärrechtlich etabliert. ${ }^{373} \mathrm{Da}$ die Charta jedoch jünger ist als die Datenschutzrichtlinie, kann Satz 2 auch als primärrechtliche Bestätigung von Art. 12 lit. a DSRL gelesen werden. ${ }^{374}$

Der Europäische Gerichtshof hat festgestellt, dass es zur Wahrung des Auskunftsrechts nach Art. 8 Abs. 2 Satz 2 GRCh genügt, dass der Antragsteller „eine vollständige Übersicht dieser Daten in verständlicher Form erhält, d.h. in einer Form, die es ihm ermöglicht, von diesen Daten Kenntnis zu erlangen und zu prüfen, ob sie richtig sind und der RL gemäß verarbeitet werden, sodass er ggf. die ihm in der RL verliehenen Rechte ausüben kann. "375

Eine Einschränkung des Auskunftsrechts ist möglich nach der allgemeinen Einschränkungsklausel des Art. 52 Abs. 1 GRCh. Die Einschränkung muss gesetzlich vorgesehen sein und den Wesensgehalt der Rechte und Freiheiten der Charta achten. Sie muss verhältnismäßig und erforderlich bezogen auf die Ziele des Gemeinwohls und den Schutz der Rechte und Freiheiten anderer sein.

Ein Ausschluss des Rechts auf Auskunft kommt aufgrund seiner zentralen Bedeutung „nur bei zwingenden und sehr gewichtigen Gemeinwohlgründen in Betracht". ${ }^{376}$

Solche zwingenden und sehr gewichtigen Gemeinwohlgründe können in Grundrechten Dritter bestehen. Hier geht es aber um den Schutz von Grundrechten der betroffenen Person selbst. Als solche Grundrechte kommen das Grundrecht auf körperliche und geistige Unversehrtheit nach Art. 3 Abs. 1 GRCh 377 und eventuell das Grundrecht auf „Nichtwissen“ infrage, ${ }^{378}$ wenn man

\footnotetext{
372 Gebot der Genauigkeit und Verständlichkeit; Bäcker, in: Kühling/Buchner, Art. 12 Rn. 11.

373 Franck, in: Gola 2017, Art. 15 Rn. 2; Ehmann, in: Ehmann/Selmayr, Art. 15 Rn. 2.

374 Bernsdorff, in: Meyer 2014, Art. 8 Rn. 23.

375 EuGH, Urteil v. 17.7.2014 - C-141/12 und C-372/12, ECLI:EU:C:2014:2081, Rn. 60 - YS.

376 Jarass 2016, Art. 8 Rn. 16.

377 S. z.B. Jarass 2016, Art. 3 Rn. 5.

378 S. zum deutschen Verfassungsrecht im Kontext genetischer Informationen Trute, in: Roßnagel 2003, Kap. 2.5 Rn. 59ff., der dieses Recht der informationellen Selbstbestimmung und dem Persönlichkeitsrecht zuordnet. Ob dieses ohne Weiteres auf die Grundrechtecharta übertragen werden kann, bedürfte ausführlicher Untersuchungen.
} 
dieses als Konkretisierung des Grundrechts auf Menschenwürde in Art. 1 GRCh verstehen wollte. ${ }^{379}$ Diese beiden Grundrechte können mit dem Grundrecht auf Datenschutz konkurrieren, das in Art. 8 Abs. 2 Satz 2 GRCh das Grundrecht auf Auskunft über die eigenen, von anderen verarbeiteten Daten gewährleistet. Einen Ausgleich zwischen seinen eigenen Grundrechten herbeizuführen, fällt in die Entscheidungsfreiheit des Einzelnen und lässt eine gesetzliche Regelung nicht zu. Dadurch löst sich der Konflikt zwischen dem Wunsch nach Nichtwissen und dem Wunsch nach Auskunft. Hier kann der Verantwortliche nachfragen, ob die betroffene Person tatsächlich diese Auskunft möchte, die für sie belastend oder schädlich sein könnte. Die grundrechtliche Lösung kann aber nur darin bestehen, die betroffene Person diese Frage entscheiden zu lassen. In einem Konflikt zwischen Auskunft und körperliche Unversehrtheit ist die Lösung grundsätzlich auf die gleiche Weise zu suchen. Dies legt auch Art. 3 Abs. 2 lit. a GRCh nahe, der die Entscheidung in Gesundheitsfragen der betroffenen Person überlässt und die freie Einwilligung des Betroffenen nach vorheriger Aufklärung zur Voraussetzung jedes Heileingriffs macht. Lediglich wenn die betroffene Person objektiv nicht in der Lage ist, diese Entscheidung verständig zu treffen, könnte eine Verweigerung der Auskunft trotz Antrags der betroffenen Person vorgesehen werden. Fraglich ist, ob eine solche Ausnahme des Auskunftsanspruchs bereits dem geltenden Recht entnommen werden kann oder ob ein Gesetzgeber eine solche Ausnahme einführen kann.

\subsection{Beschränkung der Auskunft nach $§ 27$ Abs. 2 BDSG}

\27 Abs. 2 BDSG-neu enthält zwei Möglichkeiten zur Beschränkung des Rechts auf Auskunft nach Art. 15 DSGVO.

Das Auskunftsrecht aus Art. 15 DSGVO ist nach Maßgabe von $\mathbb{2 7}$ Abs. 2 Satz 1 BDSG-neu insoweit beschränkt, als diese Rechte voraussichtlich die Verwirklichung der Forschungs- oder Statistikzwecke unmöglich machen oder ernsthaft beeinträchtigen und die Beschränkung für die Erfüllung der Forschungsoder Statistikzwecke notwendig ist. $\mathbb{} 27$ Abs. 2 Satz 1 BDSG-neu ist dabei sprachlich eng an Art. 89 Abs. 2 DSGVO angelehnt.

$\mathbb{\$} 27$ Abs. 2 Satz 2 BDSG-neu enthält eine weitere Einschränkung des Auskunftsrechts. Danach besteht das Auskunftsrecht nach Art. 15 DSGVO nicht, „wenn die Daten für Zwecke der wissenschaftlichen Forschung erforderlich sind und die Auskunftserteilung einen unverhältnismäßigen Aufwand erfordern würde“.

379 Dies ist bisher allerdings weder von der Rechtsprechung des EuGH noch von der Kommentarliteratur zur GRCh erfolgt. 
Wie bereits oben festgestellt, versucht der Gesetzgeber mit dieser Regelung $\mathbb{} 33$ Abs. 2 Satz 1 Nr. 5 i.V.m. $\mathbb{} 34$ Abs. 7 sowie $\mathbb{S} 19 a$ Abs. 2 Nr. 2 BDSG a.F. in das neue Bundesdatenschutzgesetz zu übertragen. ${ }^{380}$ Er beruft sich dabei auf Art. 23 Abs. 1 lit. i DSGVO. ${ }^{381}$

Auf verfassungsrechtlicher Ebene ergibt sich das Recht auf Auskunft aus dem Recht auf informationelle Selbstbestimmung. Bezogen auf Krankenunterlagen etwa hat das Bundesverfassungsgericht festgestellt, dass sich ein Anspruch auf Einsicht aus dem Recht auf Selbstbestimmung und der personalen Würde des Patienten ergibt. ${ }^{382}$ Es zählt zu den verfahrensrechtlichen Schutzvorkehrungen des Rechts auf informationelle Selbstbestimmung. ${ }^{383}$ Aufgrund der verfassungsrechtlichen Dimension des Auskunftsrechts sind seinen Beschränkungen hohe Hürden gesetzt. Eine Beschränkung ist „allenfalls aus triftigen Gemeinwohlgründen oder zum Schutze Dritter“ möglich. ${ }^{384}$ Eine Auskunftsbeschränkung zum Wohl der betroffenen Person ist damit aus verfassungsrechtlicher Sicht bei Beachtung der hohen Hürden grundsätzlich möglich.

\subsection{Beschränkung der Auskunftserteilung zum Wohl der betroffenen Person}

Nach Erwägungsgrund 2 DSGVO soll die Datenschutz-Grundverordnung zum Wohlergehen natürlicher Personen beitragen. Das Wohl des Betroffenen wird sonst an keiner Stelle in der Verordnung explizit angesprochen.

Fraglich ist, ob ein Ausschluss der Auskunftserteilung nach Art. 15 DSGVO auch aus therapeutischen sowie ethischen Erwägungsgründen zum Wohl der betroffenen Person möglich ist. Der Bundesrat hatte im Rahmen des Gesetzgebungsverfahrens zum Gesetz zur Anpassung des Datenschutzrechts an die Verordnung (EU) 2016/679 und zur Umsetzung der Richtlinie (EU) 2016/680 darum gebeten, zu prüfen, ob ein solcher Ausschluss Aufnahme in das Gesetz finden sollte. ${ }^{385}$ Die Bundesregierung hatte angekündigt, „die Vorschläge des Bundesrates im Bereich von Wissenschaft und Forschung im weiteren Verfahren“ zu prüfen. ${ }^{386}$ Sie hat den Vorschlag aber letztlich nicht übernommen.

\footnotetext{
380 BT-Drs. $18 / 11325$, S. 99.

381 BT-Drs. 18/11325, S. 99. A.A. Johannes/Richter, DuD 2017, 300 (303): § 27 Abs. 2 Satz 2 BDSG-neu genüge den Anforderungen von Art. 23 Abs. 2 DSGVO nicht, könne aber auf Art. 89 Abs. 2 DSGVO gestützt werden.

382 BVerfG, NJW 1999, 1777.

383 Di Fabio, in: Mainz/Dürig 2016, Art. 2 Rn. 178.

384 Di Fabio, in: Mainz/Dürig 2016, Art. 2 Rn. 178.

385 BR-Drs. 110/17 (B) vom 10. März 2017, 25, Nr. 27 lit. d: „Der Bundesrat bittet im weiteren Gesetzgebungsverfahren zu prüfen, inwieweit ein Ausschluss der Auskunftserteilung neben den in $§ 27$ BDSG-E genannten Voraussetzungen nach objektiven Kriterien auch aus therapeutischen sowie ethischen Erwägungsgründen zum Wohl der betroffenen Person aufgenommen werden sollte."

386 Gegenäußerung der Bundesregierung, BT-Drs. 19/11655, 53.
} 


\subsubsection{Beschränkung zum Wohl Dritter}

Möglich ist ein Ausschluss des Rechts aus Art. 15 Abs. 3 DSGVO auf Bereitstellung einer Kopie der personenbezogenen Daten, die Gegenstand der Verarbeitung sind. Dieses darf nach Art. 15 Abs. 4 DSGVO die Rechte und Freiheiten anderer Personen nicht beeinträchtigen. Damit ist zunächst eine Beschränkung des Auskunftsanspruchs aus therapeutischen und ethischen Erwägungsgründen zum Wohl Dritter möglich.

Darüber hinaus ist eine Beschränkung des Auskunftsrechts nach Art. 23 Abs. 1 lit. i Alt. 2 DSGVO zum Schutz der Rechte und Freiheiten anderer Personen möglich.

\subsubsection{Beschränkung zum Wohle des Betroffenen}

Direkt aus Art. 15 DSGVO ergibt sich jedoch keine mit Art. 15 Abs. 4 DSGVO vergleichbare Beschränkung zum Wohl der betroffenen Person selbst. Art. 15 DSGVO kann aber nach Maßgabe von Art. 23 Abs. 1 DSGVO durch Rechtsvorschrift der Union oder eines Mitgliedstaats ${ }^{38}$ eingeschränkt werden. Jede Beschränkung muss dabei den Wesensgehalt der Grundrechte und Grundfreiheiten achten und eine in einer demokratischen Gesellschaft notwendige und verhältnismäßige Maßnahme darstellen. Dies entspricht im Wesentlichen Art. 52 Abs. 1 GRCh. Darüber hinaus enthält Art. 23 Abs. 1 DSGVO einen Katalog von Zielen, die eine Beschränkung grundsätzlich rechtfertigen können. Die Voraussetzungen des Art. 23 DSGVO müssen kumulativ vorliegen. ${ }^{888}$ Der Katalog der Ziele, die eine Beschränkung rechtfertigen können, ist abschließend.

Zusätzlich sind die Vorgaben zu beachten, die der Europäische Gerichtshof in seiner Rechtsprechung entwickelt hat. ${ }^{389}$ Diese entsprechen letztlich dem, was auch die Verordnung selbst fordert:

- Klarheit und Präzision der beschränkenden Vorschrift,

- Garantien für den Schutz der Rechte des Betroffenen,

- Achtung des Wesensgehalts der relevanten Crundrechte und

- Notwendigkeit der Einschränkung.

Bezogen auf die Ausgangsfrage kommt hier primär Art. 23 Abs. 1 lit. i DSGVO in seiner ersten Alternative in Betracht. Danach ist eine Beschränkung unter den genannten Voraussetzungen möglich, sofern diese den Schutz der betroffenen Person sicherstellt. Diese Voraussetzung ist äußerst weit gefasst und entspricht wörtlich der Regelung des Art. 13 Abs. 1 lit. g DSRL. Aber auch die in den Buchstaben a bis h und $\mathrm{j}$ genannten Zielen dienen letztlich dem Wohl

387 Bezogen auf die Gesetzgebungsmaßnahme sind zusätzlich die Vorgaben von Art. 23 Abs. 2 DSGV0 zu beachten.

388 Bertermann, in: Ehmann/Selmayr, Art. 23 Rn. 3.

389 S. insbesondere EuGH, Urteil vom 6.10.2015, C-362/14. ECLI:EU:C:2015:650, Schrems. 
der betroffenen Person. Auf grundrechtlicher Ebene findet Art. 23 Abs. 1 lit. i DSGVO seine Rechtfertigung unter anderem in Art. 3 Abs. 1 GRCh.

Zur Vorgängerregelung in Art. 13 Abs. 1 lit. g DSRL wurde gefordert, die Beschränkung dürfe nicht dazu führen, dass „unter dem Gesichtspunkt des Betroffenenschutzes dessen grundrechtliche Position ad absurdum geführt wird“. ${ }^{390}$ Dies ist auch für Art. 23 Abs. 1 lit. i DSGVO zu fordern. Ein auch im Kontext von Art. 23 DSGVO relevantes Beispiel liefert Erwägungsgrund 42 DSRL, wo es heißt, die Mitgliedstaaten können das Auskunftsrecht im Interesse der betroffenen Person in der Art beschränken, dass „Auskunft über medizinische Daten nur über ärztliches Personal erhalten werden kann“.

\subsubsection{Beschränkung nach $\S 630 \mathrm{~B}$ BGB}

Ein Beispiel für eine mit Art. 23 Abs. 1 lit. i DSGVO kompatible Beschränkung stellt bereits jetzt $\$ 630$ g BGB dar. Dieser enthält in $\$$ 630g Abs. 1 BGB ein Recht auf unverzügliche Einsichtnahme in die Patientenakte im Kontext von Behandlungsverträgen i.S.v. $\$ 630 a$ BCB. Dieses wird jedoch nur gewährt, „soweit der Einsichtnahme nicht erhebliche therapeutische Gründe entgegenstehen“. ${ }^{391}$ Die Ablehnung ist zu begründen. ${ }^{392}$ Umfasst vom Einsichtsrecht des Patienten sind auch „Niederschriften über persönliche Eindrücke und subjektive Wahrnehmungen des Behandelnden“. ${ }^{393}$ Erhebliche therapeutische Gründe sollen etwa dann vorliegen, wenn die Ausübung des Rechts auf Einsichtnahme zu einer „erheblichen gesundheitlichen (Selbst-)Schädigung des Patienten" führen könnte. ${ }^{394}$ Sie sind sorgfältig für jeden Einzelfall zu ermitteln und auf konkrete und substantiierte Anhaltspunkte zu stützen. ${ }^{395}$ Im Zweifel ist für die Auskunft zu entscheiden. ${ }^{396}$ Eine paternalistische Bevormundung des Patienten durch den Behandelnden ist ausdrücklich nicht erwünscht. ${ }^{397}$

Nach $\mathbb{5} 630$ g Abs. 1 Satz 3 BGB ist $\$ 811$ BGB anzuwenden. Dieser besagt, dass die Vorlegung der Patientenakte an dem Ort zu erfolgen hat, an welchem sie sich befindet. ${ }^{398}$ Ausnahmen von diesem Grundsatz sind im Einzelfall aber möglich.

390 Ehmann/Helfrich 1999, Art. 13 Rn. 76.

$391 § 630 \mathrm{~g}$ Abs. 1 Satz 1 BGB.

$392 \$ 630 \mathrm{~g}$ Abs. 1 Satz 2 BGB.

393 BT-Drs. 17/10488, 27. Das Persönlichkeitsrecht des Behandelnden soll nur in Ausnahmefällen einen Ausschluss rechtfertigen können; Wagner, in: MüKo BGB, \630g Rn. 8.

394 BT-Drs. 17/10488, 26. Dies soll etwa dann der Fall sein, wenn der Patient durch die Einsichtnahme „zum Selbstmord veranlasst würde“; BVerwGE 82, 45. Die Schwelle ist also hoch angesetzt.

395 BT-Drs. 17/10488, $26 \mathrm{f}$.

396 Wagner, in: MüKo BGB, \630g Rn. 10.

397 BT-Drs. 17/10488, 26; Wagner, in: MüKo BGB, \$630g Rn. 11.

398 Ein Ortswechsel kann nur aus „wichtigem Grund“ verlangt werden; $\$ 811$ Abs. 1 Satz 2 BGB. Ein solcher soll etwa „bei einer nicht unerheblichen Erkrankung des Patienten“ oder bei einem Umzug des Behandelnden“ vorliegen; BT-Drs. 17/10488, 27. 
Anzumerken ist, dass vor einer vollständigen Verweigerung der Einsichtnahme eine partielle Verweigerung als milderes Mittel zu prüfen ist. 399 Zudem kommen die „durch den Behandelnden unterstützte oder auch begleitende Einsichtnahme“ oder die Vermittlung durch eine dritte Person ebenfalls als milderes Mittel in Betracht. ${ }^{400}$ Ferner ist der Rechtsgedanke des $\mathbb{6} 603 \mathrm{e}$ Abs. 5 BGB zu beachten, wonach auf den Entwicklungsstand und die Verständnismöglichkeit sowie auf das Wohl des Patienten Rücksicht zu nehmen ist. ${ }^{401}$ Eine vollständige Verweigerung der Einsichtnahme dürfte damit auf wenige Extremfälle beschränkt sein. ${ }^{402}$

$\mathrm{Zu}$ dem Recht auf unverzügliche Einsichtnahme in die Patientenakte aus $\$ 630$ Abs. 1 BCB tritt in $₫ 630$ Abs. 2 Satz 1 BCB ein Recht auf Erhalt physischer oder elektronischer Abschriften der Patientenakte. Hier gelten dieselben Einschränkungsmöglichkeiten wie im Falle der Einsichtnahme. Der Anspruch auf eine bestimmte Form der Abschriften soll jedoch auf Fälle beschränkt sein, in denen der Patient ein berechtigtes Interesse an dieser hat. ${ }^{003}$

$\$ 630$ Abs. 3 BGB enthält Regelungen zur Wahrnehmung der Rechte aus Abs. 1 und $2 \mathrm{im}$ Falle des Todes des Patienten durch Erben und nächste Angehörige.

Das Auskunftsrecht nach $\$ 630 g$ BCB kann durch individuelle Parteivereinbarung, nicht aber durch allgemeine Geschäftsbedingungen ausgeschlossen werden. ${ }^{404}$

Es zeigt sich mit Blick auf $₫ 630$ g BCB, dass der Einschränkung der Auskunftserteilung aus therapeutischen und ethischen Erwägungsgründen zum Wohl der betroffenen Person im Falle von Gesundheitsdaten hohe Hürden gesetzt sind. Jedes Ersuchen ist als individueller Einzelfall umfassend zu prüfen, sofern tatsächlich eine Ablehnung in Betracht kommt. Insbesondere kann nicht jede potenzielle Schädigung des Betroffenen durch die Einsichtnahme als Rechtfertigungsgrund dienen; diese muss vielmehr erheblich sein und etwa der Suizid des Betroffenen drohen. Im Extremfall kann sich ein Schädigungspotenzial sogar zu einem Beschränkungsgebot verdichten.

Die verfassungsrechtlichen Grundlagen für $\mathbb{6} 630 \mathrm{~g}$ BCB hat das Bundesverfassungsgericht 2006 in einem Beschluss aufgearbeitet. ${ }^{405}$ Das Recht aus $\mathbb{5} 630 \mathrm{~g}$ BGB dient vornehmlich dem Recht des Patienten auf informationelle Selbstbestimmung. ${ }^{406}$ Die vom Gericht aufgearbeiteten Grundlagen lassen sich abstrahieren und auf andere Sachverhalte übertragen. Auf diese Weise wurden

399 So der Umkehrschluss aus BT-Drs. 17/10488, 26.

400 BT-Drs. 17/10488, 27.

401 Wagner, in: MüKo BGB, \630g Rn. 5.

402 Sie ist allenfalls ultima ratio; Wagner, in: MüKo BGB, \$630g Rn. 12.

403 Wagner, in: MüKo BGB, \630g Rn. 20.

404 Wagner, in: MüKo BGB, \$ $630 \mathrm{~g}$ Rn. 30.

405 BVerfG, Beschluss vom 9.1.2006 - 2 BvR 443/02, NJW 2006, 1116.

406 BT-Drs. 17/10488, 26; Wagner, in: MüKo BGB, \ 630 g Rn. 4. 
bereits auf nationaler Ebene Einsichtsrechte in Pflegeunterlagen ${ }^{407}$ und in die im Kontext der Teilnahme an einer Lehranalyse angefertigten Dokumentation ${ }^{408}$ anerkannt. ${ }^{409}$ Auch eine Übertragung auf das Auskunftsrecht aus Art. 8 GRCh ist möglich, soweit dieses dem aus dem Recht auf informationelle Selbstbestimmung abgeleiteten Recht wesensgleich ist. Insofern kann $\mathbb{5} 630$ g BGB als eine mit Art. 23 Abs. 1 lit. i DSGVO vereinbare Ausfüllung der Öffnungsklausel angesehen werden.

\subsubsection{Beschränkung im Kontext wissenschaftlicher Forschung}

Fraglich ist, ob eine solche Beschränkung auch im Kontext von wissenschaftlichen Forschungsvorhaben möglich wäre.

Die Datenschutz-Grundverordnung will die Verarbeitung von personenbezogenen Daten im wissenschaftlichen Kontext bevorzugen und enthält deshalb mit Art. 85 Abs. 2 DSGVO eine Öffnungsklausel, die im Vergleich zu Art. 23 Abs. 1 DSGVO deutlich weitreichendere ${ }^{410}$ Abweichungen und Ausnahmen von den Vorgaben der Verordnung durch die Mitgliedstaaten ermöglicht. Eine Einengung findet aber wiederum durch die Verwendung des Begriffspaars „academic expression" statt. ${ }^{41}$ Hier steht die Meinungsäußerung im Vordergrund. Eine Beschränkung des Auskunftsrechts zu wissenschaftlichen Forschungszwecken kann deshalb nicht auf Art. 85 Abs. 2 DSGVO gestützt werden.

Infrage käme aber Art. 89 Abs. 2 DSGVO, der zwar Ausnahmen von den Rechten aus Art. 15 DSGVO ermöglicht, dies aber nur insoweit, „als diese Rechte voraussichtlich die Verwirklichung der spezifischen Zwecke unmöglich machen oder ernsthaft beeinträchtigen und solche Ausnahmen für die Erfüllung dieser Zwecke notwendig sind“. Dies ist bei einer Beschränkung der Auskunftserteilung aus therapeutischen und ethischen Erwägungsgründen zum Wohl der betroffenen Person nicht der Fall. Eine derartige Beschränkung muss deshalb auf Art. 23 Abs. 1 DSGVO gestützt werden und kann nicht an der Bevorzugung der wissenschaftlichen Forschung teilhaben.

Eine Beschränkung wäre aber nach Art. 23 Abs. 1 lit. i DSGVO möglich, sofern die Hürden entsprechend hoch angesetzt werden und die Beschränkung als Ultima Ratio ausgestaltet wäre. ${ }^{412}$ Da auch hier auf erhebliche Schäden des Betroffenen durch die Offenlegung ihm selbst gegenüber abzustellen ist, dürfte eine solche Beschränkung faktisch nur auf Gesundheitsdaten Anwendung

407 BGHZ 185, 74.

408 BGH, Urteil vom 7.11.2013 - III ZR 54/13.

409 S. Wagner, in: MüKo BGB, $\$ 630 \mathrm{~g}$ Rn. $31 \mathrm{f}$.

410 Pötters, in: Gola 2017, Art. 85 Rn. 16.

411 S. Kap. 4.2.

412 S. auch Bäcker, in: Kühling/Buchner, Art, 23 Rn. 30, der Art. 23 Abs. 1 lit. Alt. 1 DSGV0 „allenfalls einen äußerst schmalen Anwendungsbereich" zugesteht. 
finden. Unabhängig davon kann das Auskunftsrecht aber auch im Kontext wissenschaftlicher Forschung durch Individualvereinbarung ausgeschlossen werden. ${ }^{413}$

Eine Beschränkung der Auskunftserteilung aus therapeutischen und ethischen Erwägungsgründen zum Wohl der betroffenen Person im Kontext von wissenschaftlichen Forschungsvorhaben ist also durchaus denkbar, müsste aber auf einer konkreten Rechtsvorschrift beruhen. Eine solche Rechtsvorschrift enthält das neue Bundesdatenschutzgesetz nicht. Sie könnte mit Blick auf die verfassungsrechtlichen und unionsrechtlichen Vorgaben allerdings geschaffen werden. Da Einschränkungen des Auskunftsrechts nach Art. 23 Abs. 1 DSGVO nur im Wege von Gesetzgebungsmaßnahmen möglich sind, wäre eine entsprechende Rechtsvorschrift auch angezeigt. Der Begriff der Gesetzgebungsmaßnahme ist vor dem Hintergrund von Art. 52 Abs. $1 \mathrm{CRCh}^{414}$ jedoch weit auszulegen, sodass er etwa auch Richterrecht umfasst; ein formelles Gesetz ist nicht erforderlich. ${ }^{415}$

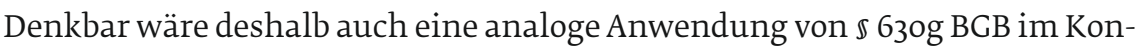
text wissenschaftlicher Forschungsvorhaben. Dies gründet darauf, dass $₫ 6309$ BCB nicht als abschließende Regelung konzipiert ist und nicht auf die Akteneinsicht im Rahmen von Behandlungsverträgen beschränkt ist. ${ }^{416}$ Eine analoge Anwendung des $₫ 630 g$ BGB findet etwa bezogen auf die Pflegedokumentation statt. Zudem ist eine analoge Anwendung auf Lehranalysen anerkannt. ${ }^{417}$

\subsection{Ergebnis zum Ausschluss der Auskunftserteilung zum Wohl der betroffenen Person}

Das Recht auf Auskunft in Art. 8 Abs. 2 Satz 2 GRCh und Art. 15 DSGVO ist im Wesentlichen deckungsgleich mit seinem Vorgänger aus der Datenschutzrichtlinie und seiner Umsetzung im bisherigen Bundesdatenschutzgesetz. Es bildet die Basis für die Überprüfung der Rechtmäßigkeit der Verarbeitung durch den Betroffenen. Beschränkungen des Auskunftsrechts - auch zum Wohl der betroffenen Person - sind nach Art. 52 GRCh und Art. 23 DSGVO möglich, aber in besonderem Maße begründungsbedürftig. Beschränkungsmöglichkeiten für das Recht auf Auskunft enthält etwa $\$ 27$ Abs. 2 BDSG-neu, die aber für eine Verweigerung der Auskunftserteilung zum Wohl der betroffenen Person nicht gelten.

413 Zu Einschränkungsmöglichkeiten von Rechten der betroffenen Person s. Kap. 3.4.3

414 Borowsky, in: Meyer, Art. 52 Rn. $20 f$.

415 S. auch Kühling/Martini u.a. 2016, 72; Bäcker, in: Kühling/Buchner, Art. 23 Rn. 35.

416 Wagner, in: MüKo BGB, \$630g Rn. 31.

417 S. Mansel, in: Jauernig, $\$ 630$ BGB, Rn. 2. S. auch bereits oben in Kap. 5.4.3. 
Ein Ausschluss der Auskunftserteilung zum Wohl der betroffenen Person ist

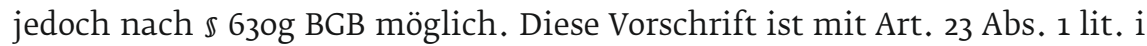
DSGVO kompatibel. Dem Ausschluss sind dabei hohe Hürden gesetzt.

Auch im Kontext von wissenschaftlichen Forschungsvorhaben ist eine ähnliche Einschränkung - als Ultima Ratio - grundsätzlich möglich. Diese müsste aber auf einer konkreten Rechtsvorschrift beruhen. Eine solche Rechtsvorschrift existiert derzeit nicht, könnte aber geschaffen werden. Die Beschränkung muss aber auf Art. 23 Abs. 1 DSGVO gestützt werden und kann nicht an der Bevorzugung der wissenschaftlichen Forschung durch die Grundverordnung teilhaben. Unabhängig davon kann das Auskunftsrecht aber auch im Kontext wissenschaftlicher Forschung durch Individualvereinbarung ausgeschlossen werden. 